\title{
Link Breakage Time Prediction Algorithm for Efficient Power and Routing in Unmanned Aerial Vehicle Communication Networks
}

\author{
Haque Nawaz ${ }^{1}$ \\ Dept. Computer Science \\ SZABIST Karachi \\ Sindh Madressatul Islam University \\ Karachi, Sindh, Pakistan
}

\author{
Husnain Mansoor $\mathrm{Ali}^{2}$ \\ Dept. Computer Science \\ Shaheed Zulfikar Ali Bhutto Institute of \\ Science and Technology \\ Karachi, Sindh, Pakistan
}

\begin{abstract}
UAV Communication Networks (UAVCN) comes under the umbrella of Ad hoc Network technology. It has the critical differences with existing wireless networks, which are high mobility, high speed, dynamic updates, and changes in topology due to high movement, which creates the problem of link breakages and affects the routing performance. This problem degrades the performance of UAVCN in terms; it decreases throughput and minimizes the packet delivery ratio. In this paper, we have tried to overcome this problem by considering the received signal power strength (RSPS). We have proposed an algorithm which uses the received signal power strength and time and calculates the link breakage time prediction by using the interpolation method. We have implemented the proposed technique by modifying the OLSR protocol. The extended protocol termed EPOLSR, which efficiently using the signal power strength and time and increasing the performance of UAVCN. The extended protocol implemented by using a research tool network simulator (v3). The metrics received rate, no of received packets, throughput, and packet delivery ratio (PDR) is considered for evaluation. We have examined the proposed EPOLSR with existing routing protocols. It has been observed that the modified protocol performs better concerning all existing evaluated routing approaches.
\end{abstract}

Keywords-UAV; link breakage; algorithm; power; RSPS; routing

\section{INTRODUCTION}

In this study, we have explored the emerging area of adhoc networks, which is known as UAVCN. These nodes can be deployed for a specific operation; either it belongs to the military or civilian mission. [1,2]. These network nodes have high movement. However, it changes the frequent topology; that's why link breakages issue affects the routing [3]. The coverage increased by minimizing the interference of UAVs' communication [4]. The environment and terrain affect the UAVs' communication, hence to overcome these obstacles, a hybrid mechanism of unicast and geocast routing used to know the trajectory and location [5]. The authors optimized the route by using the neural network concepts and implemented the Dynamic Source Routing (DSR) protocol for evaluation and optimization [6]. The researchers proposed the hybrid protocol which helps in link establishment [7]. In this study, experimental work carried out by comparing the adhoc on demand routing (AODV), DSDV, and OLSR routing protocol [8].

In this paper, we have organized the work into sections. The first section represents the introduction, and the second section provides the information of OLSR working mechanism, the third section contains a modification of OLSR Hello and Topology Messages by updating the reserved field, the fourth section indicates the prediction of route failure and Link breakage prediction. The fifth section presents the proposed algorithm. The sixth section highlights the research methodology. The seventh section describes the Results, and in the Eighth section, we have concluded.

\section{THE OLSR WORKING MECHANISM}

It is the proactive routing approach that operates two nodes by sending Hello Messages. It discovers the neighbors and maintains the neighbors' table. It updates the topology status and updates the topology information and maintains the routing table. Also, share the MID messages. The neighbor discovery process is mentioned as:

Fig. 1 shows the neighbor discovery process through Hello Messages. The $\mathrm{X}$ node forwards an empty message Hello. The $\mathrm{Y}$ node receives the message and stores information $\mathrm{X}$ as an asymmetric neighbor because the $\mathrm{Y}$ address is not available in Hello message. Y node then forwards Hello Message by asserting $\mathrm{X}$ is an asymmetric neighbor as soon as $\mathrm{X}$ obtains this message and gets its information address and then declares $Y$ as neighbor symmetric. In last, $\mathrm{X}$ node incorporates $\mathrm{Y}$ in the Hello, it forwards, and $\mathrm{Y}$ recognize $\mathrm{X}$ as neighbor symmetric as receiving Hello message.

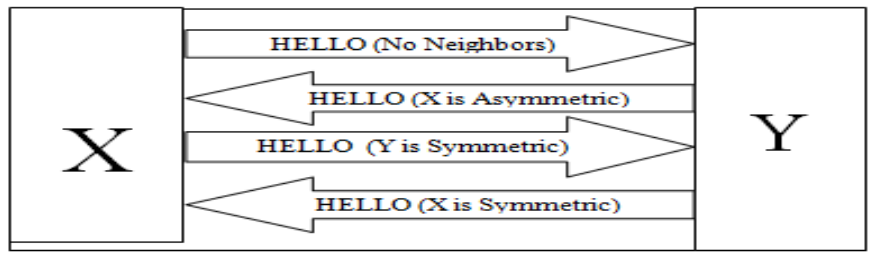

Fig. 1. Neighbor Discovery Process through Hello Messages. 


\begin{tabular}{|c|c|c|c|}
\hline \multicolumn{2}{|c|}{ Reserved } & Htime & Willingness \\
\hline Linkcode & Reserved & Link Message service \\
\hline \multicolumn{3}{|c|}{ Neighbor Interface Address } \\
\hline \multicolumn{3}{|c|}{ Neighbor Interface Address } \\
\hline
\end{tabular}

Fig. 2. Hello Message Format of OLSR.

The above Fig. 2 shows the Hello Message Format of OLSR. According to RFC 3626, the OLSR furthermore extended or modified by using the reserved field. However, Htime shows the time of the next Hello packet before transmission. The willingness field represents that the willingness of the node when it forwards the traffic. The sender and the neighbor node-link information consists of the link code. It declares the status information of the neighbor node. Link message size provides the total link messages length. The neighbor interface address knows the interface of the neighbor node.

Fig. 3 shows the Topology Control Message Format of OLSR. The Advertised Neighbor Sequence Number (ANSN) shows the incremented sequence number at any time when changes take place in the neighbor set. According to RFC 3626 , the OLSR TC packet can furthermore be extended or modified by using the reserved field. The advertised Neighbor Main Address field consists of neighbor node main addresses.

\begin{tabular}{|c|c|}
\hline ANSN & Reserved \\
\hline Advertised Neighbor Main Address \\
\hline Advertised Neighbor Main Address \\
\hline \multicolumn{2}{c|}{$\ldots \ldots \ldots \ldots \ldots \ldots$} \\
\hline
\end{tabular}

Fig. 3. Topology Control Message Format of OLSR.

\section{MODIFICATION OF OLSR HELlO AND TOPOLOGY MESSAGES}

The OLSR hello and topology messages are furthermore extended or modified by using the reserved field. The TC packet also extended or modified by using the reserved field. We have updated these packets by adding the power information in terms of signal strength, which is shown in Fig. 4 and Fig. 5. In this study, the modified OLSR termed as EPOLSR. The objective of EPOLSR design was to improve the routing in unmanned aerial vehicle communication networks. The EPOLSR consists of the Routing Table, which stores the routes information of all reachable destination UAVs.

\begin{tabular}{|l|l|c|c|}
\hline \multicolumn{2}{|c|}{ Power } & Htime & Willingness \\
\hline Link code & Reserved & Link Message service \\
\hline \multicolumn{3}{|c|}{ Neighbor Interface Address } \\
\hline \multicolumn{3}{|c|}{ Neighbor Interface Address } \\
\hline
\end{tabular}

Fig. 4. Hello Message Format of EP OLSR.

\begin{tabular}{|c|c|}
\hline ANSN & Power \\
\hline & Advertised Neighbor Main Address \\
\hline & Advertised Neighbor Main Address \\
\hline & $\ldots \ldots \ldots \ldots \ldots \ldots$ \\
\hline
\end{tabular}

Fig. 5. Topology Control Message Format of EP OLSR.

When a node receives the Hello message, it performs the following operations.

- To populate one-hop neighbors

- To populate two-hop neighbors

- To perform MPR calculation

- To populate MPR selector set

\section{A. Neighbor Table}

The neighbor table contains the following fields.

- Node Address

- Neighbor address identification

- Power Information (LRSPS and CRSPS)

- Time Information (LRSPST and CRSPST)

- Next Link Breakage Time

Each entry in the routing table has the destination address id, next-hop address, data size in packets, delay, and next route breakage time. Based on this information, one hop table and two-hop table neighbor entries, update the routing table. Compare the topology set and update the routing table.

To improve the performance of routing, a method ought to be introduced to administer the route breakages. Although, mostly routing algorithms are used hello messages for the detection of link breakages. The proposed algorithm operates based on link breakage prediction. It estimates link breakage time based on RSPS (received signal power strength) and, it predicts route breakage time as well.

\section{Link BREAKAgE PREDICTION TIME}

The hello messages are used to uphold the prediction of link breakage time. Hence, at each time interval, a hello message is broadcasted to everyone instant neighbor of UAV. On account of RSPS intensity, the cost of subsequently, next link breakage time (y) of the analogous neighbor $\mathrm{j}$, might be enlarged or minimized. While a UAV $(\mathrm{x})$ received a hello message from another UAV (y). Then it evaluates the RSPS with the LRSPS from the same UAV. Furthermore, predicting the new cost of NLBT $(\mathrm{x}, \mathrm{y})$ using the interpolation method. The given equation (Eq. 1) computes the value of the next link breakage time $\operatorname{NLBT}(\mathrm{x}, \mathrm{y})$ :

$$
\operatorname{NLBPT}(\mathrm{x}, \mathrm{y})=\text { Crspst }+\frac{(\text { Crspst }- \text { Lrspst }) *(\text { RxThresh }- \text { Crsps })}{(\text { Crsps }- \text { Lrsps })}
$$




\section{Link BREAKAGE TIME PREDICTION ALGORITHM FOR EFFICIENT POWER AND ROUTING}

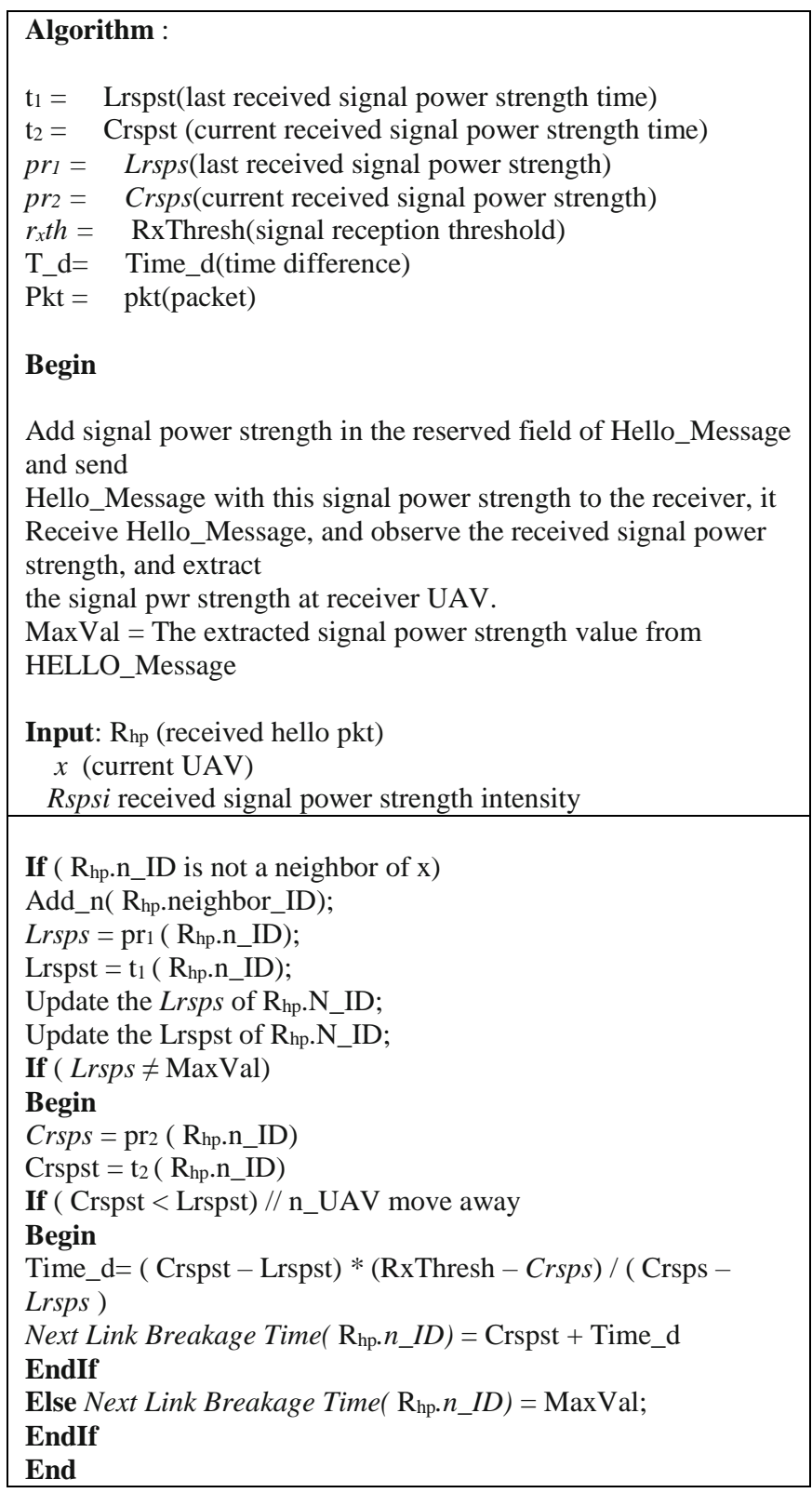

\section{RESEARCH METHODOLOGY}

The proposed approach or research methodology which we have used is the experimental method. We have used ns-3 (Network Simulator version 3. It can be used for UAVs networks simulation $[9,10]$. Using this tool, we have implemented the EP-OLSR routing protocol and compared it with OLSR, DSDV, AODV, and DSR; by using the IEEE $802.11 \mathrm{~b}$ environment. After the development of the testbed scenario, the simulation was carried out.

\section{EXPERIMENTAL WORK AND SIMULATION PARAMETERS}

In this scenario, we have used the ns- 3 simulation tool and carried out the experiment. The main characteristics of this scenario are shown in Table I.
TABLE. I. CHARACTERISTICS OF THE TESTBED SCENARIO

\begin{tabular}{|l|l|}
\hline Parameters & Values \\
\hline Simulation tool & Ns-3. \\
\hline Adhoc Routing Protocols & $\begin{array}{l}\text { EP-OLSR, OLSR, DSDV, AODV, } \\
\text { and DSR }\end{array}$ \\
\hline Network Scenario Size & $1500 x 400$ \\
\hline Number Nodes & 25 \\
\hline Data Rate & 5 Mbps \\
\hline Application & Video Streaming \\
\hline WLAN Physical Characteristics & IEEE 802.11b \\
\hline Network Protocol & IP \\
\hline Mobility model & Random Waypoint \\
\hline Scenario Simulation Time & 200 Sec \\
\hline
\end{tabular}

The scenario has been developed by using 25 nodes - the network scenario based on the area of $1000 \times 400 \mathrm{~m} 2$. The WLAN physical characteristics of standard $802.11 \mathrm{~b}$ are used. The mobility model random waypoint is used. Similarly, the EP OLSR has been developed in C++ and integrated modules in the NS-3 environment. And by using the same wireless LAN physical characteristics standard cross-layer design in the 802.11b environment and the simulation has been run. Similarly, the existing protocols, OLSR, DSDV, AODV, and DSR configured in the scenario, and the simulation has been run. The simulation runs for $200 \mathrm{~s}$. After this experimental setup, the scenario has been accomplished.

\section{SIMULATION RESULTS AND DISCUSSION}

The results show that, in the ns-3 environment, the EP OLSR Receive Rate is $38 \mathrm{Kbps}$, and on the other hand, we have observed OLSR Receive Rate is $32 \mathrm{Kbps}$, as depicted in Fig. 6. Overall, the performance of the EP OLSR protocol is found better when compared to that of OLSR.

The results show that, in the ns-3 environment, the EP OLSR Receive Rate is $38 \mathrm{Kbps}$, and on the other hand, we have observed DSDV Receive Rate is $27 \mathrm{Kbps}$, as depicted in Fig. 7. Overall, it has been found that the performance of the EP OLSR protocol is better when compared with DSDV.

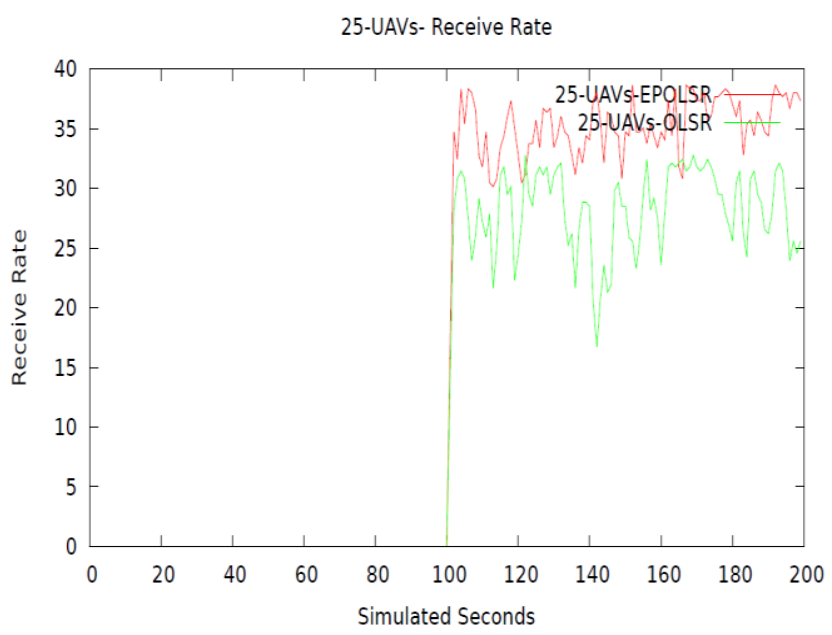

Fig. 6. 25-UAVs- Receive Rate (kbps) using EP OLSR vs. OLSR. 
25-UAVs-Receive Rate

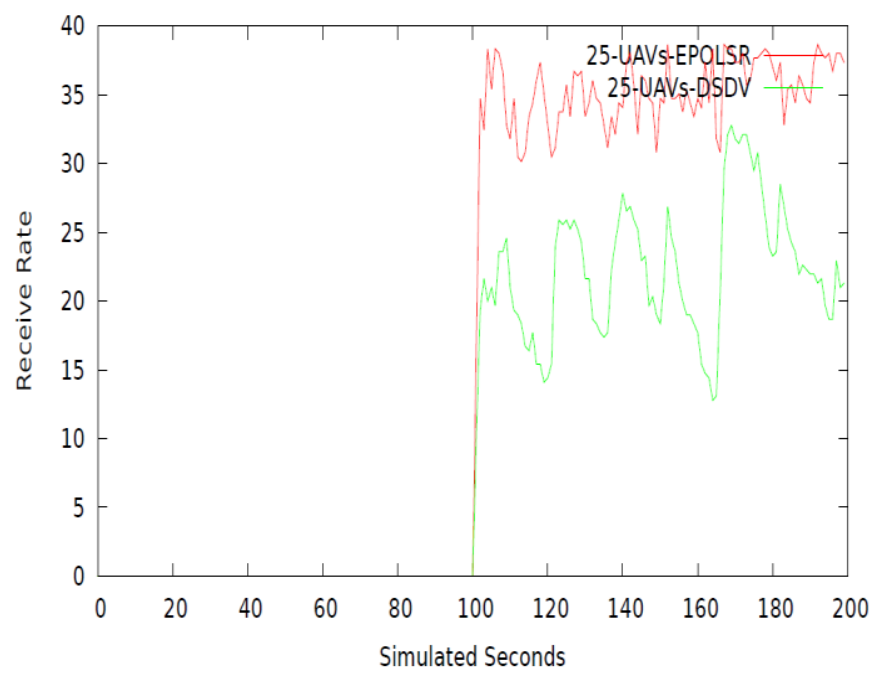

Fig. 7. 25-UAVs- Receive Rate (kbps) using EP OLSR vs. DSDV.

The results show that, in the ns-3 environment, the EP OLSR Receive Rate is $38 \mathrm{Kbps}$, and on the other hand, we have observed AODV Receive Rate is $28 \mathrm{Kbps}$, as depicted in Fig. 8. Overall, the performance of the EP OLSR protocol is better when compared with AODV.

The results show that, in the ns-3 environment, the EP OLSR Receive Rate is $38 \mathrm{Kbps}$, and on the other hand, we have observed DSR Receive Rate is $30 \mathrm{Kbps}$, as depicted in Fig. 9. Overall, the performance of the EP OLSR protocol is found better when compared to DSR.

The results show that, in the ns-3 environment, the EP OLSR Receive Rate is $38 \mathrm{Kbps}$, OLSR Receive Rate is 32 Kbps, DSDV Receive Rate is $27 \mathrm{Kbps}$, AODV No of Packets Received is $28 \mathrm{Kbps}$, on the other hand, DSR Receive Rate is $30 \mathrm{Kbps}$, as depicted in Fig. 10. Overall, it has been observed that the performance of the EP OLSR protocol is better when compared to OLSR, DSDV, AODV, and DSR.

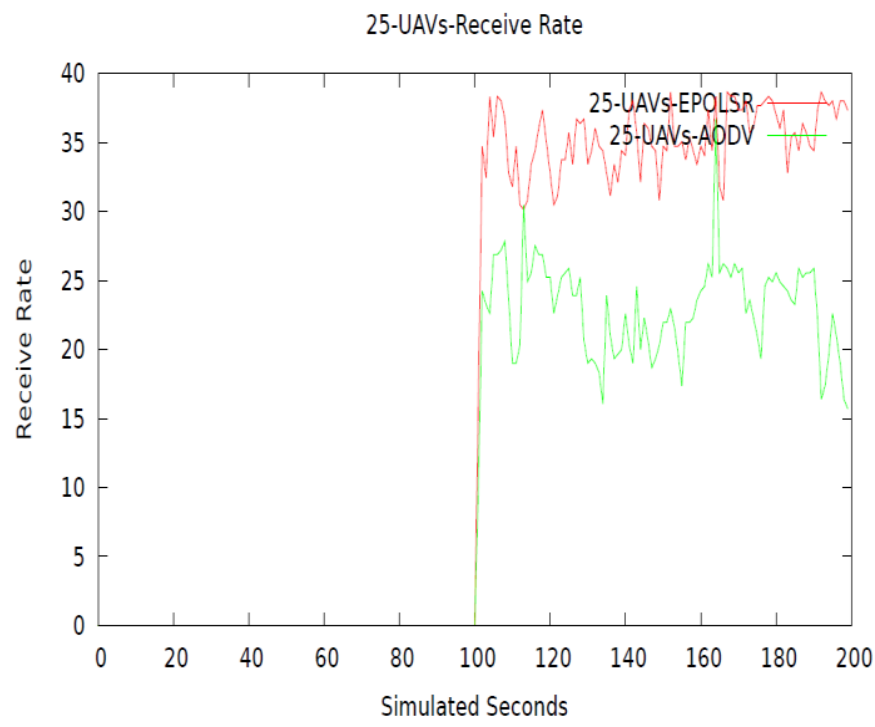

Fig. 8. 25-UAVs- Receive Rate (kbps) using EP OLSR vs. AODV.

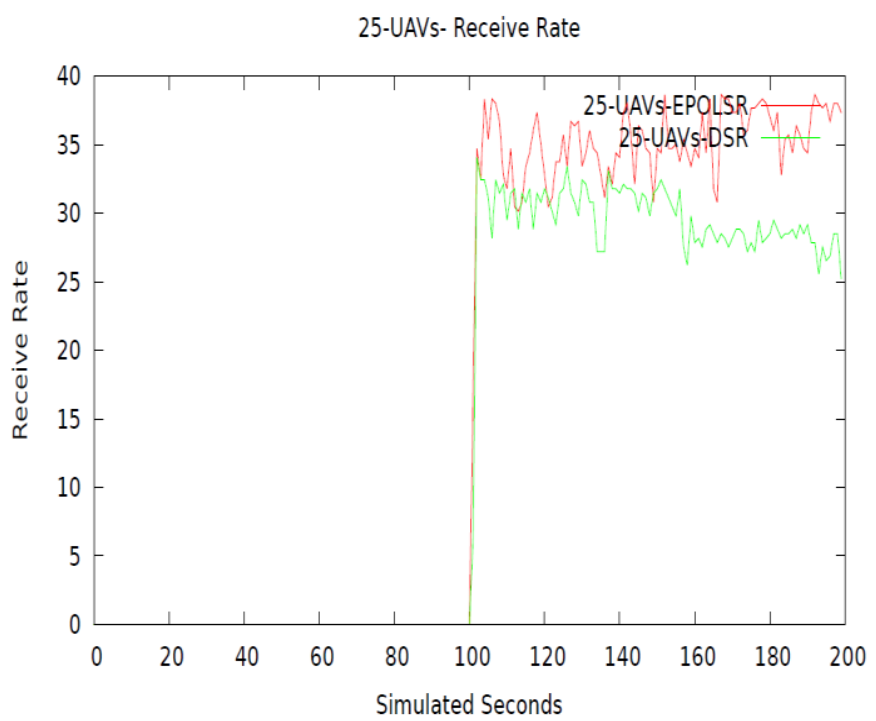

Fig. 9. 5-UAVs- Receive Rate (kbps) using EP OLSR vs. DSR.

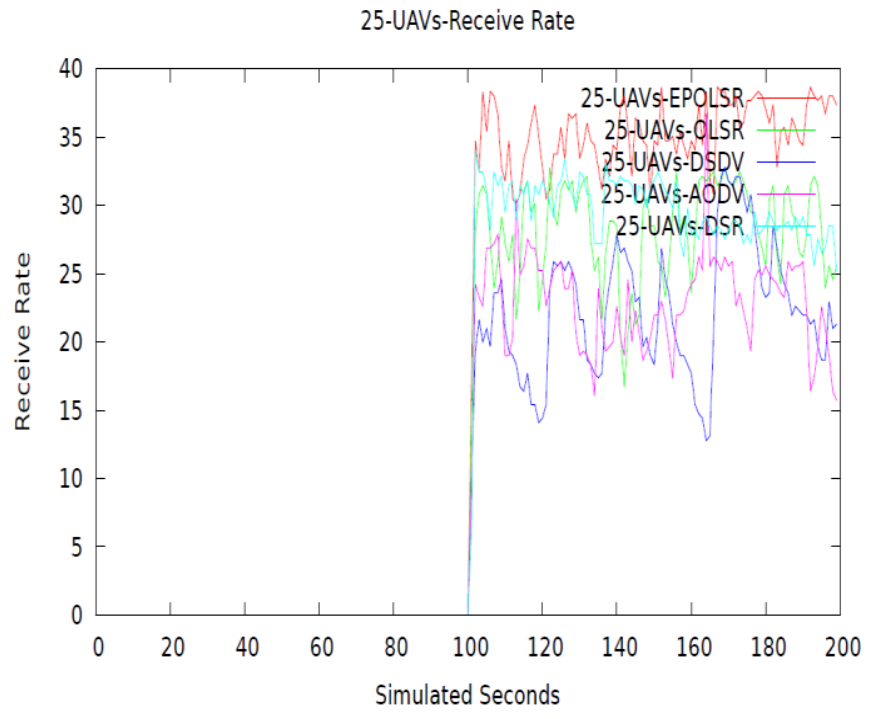

Fig. 10. 25-UAVs- Receive Rate (kbps) using EP OLSR vs. OLSR, DSDV, AODV, and DSR.

The results show that, in the ns-3 environment, the EP OLSR No of Packet Received is $38 \mathrm{Kbps}$, and on the other hand, we have observed OLSR No of Packet Received is 32 $\mathrm{Kbps}$, as depicted in Fig. 11. Overall, the performance of the EP OLSR protocol is found better when compared with OLSR.

The results show that, in the ns-3 environment, the EP OLSR No of Packet Received is $38 \mathrm{Kbps}$, and on the other hand, we have observed DSDV No of Packet Received is 26 $\mathrm{Kbps}$, as depicted in Fig. 12. Overall, the performance of the EP OLSR protocol is found better when compared with DSDV.

The results show that, in the ns-3 environment, the EP OLSR No of Packet Received is $38 \mathrm{Kbps}$, and on the other hand, we have observed AODV No of Packet Received is 26 $\mathrm{Kbps}$, as depicted in Fig. 13. Overall, the performance of the EP OLSR protocol is found better when compared with AODV. 


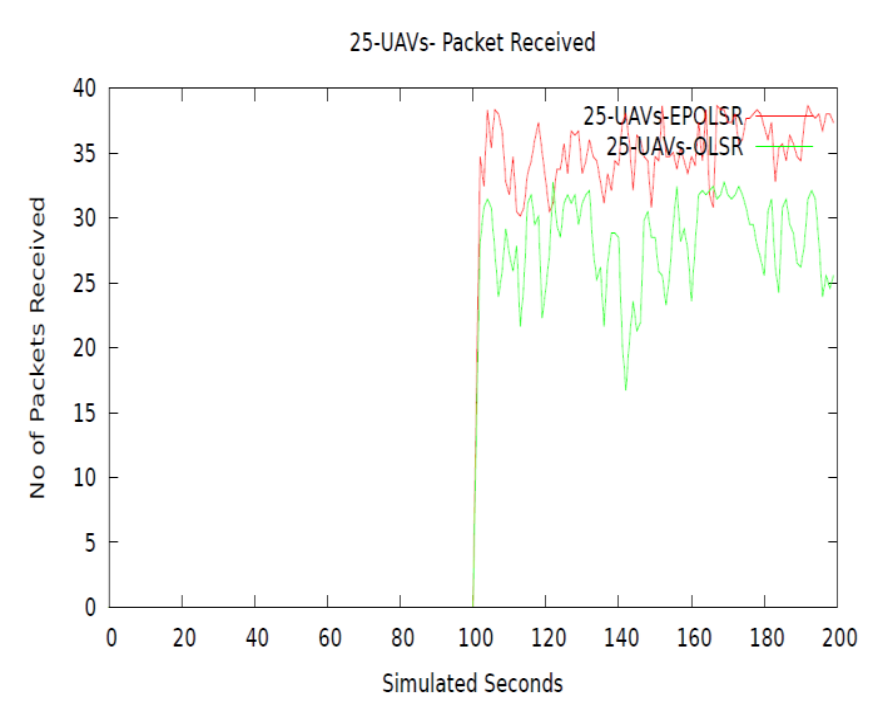

Fig. 11. 25-UAVs- No of Packet Received (kbps) using EP OLSR vs. OLSR.

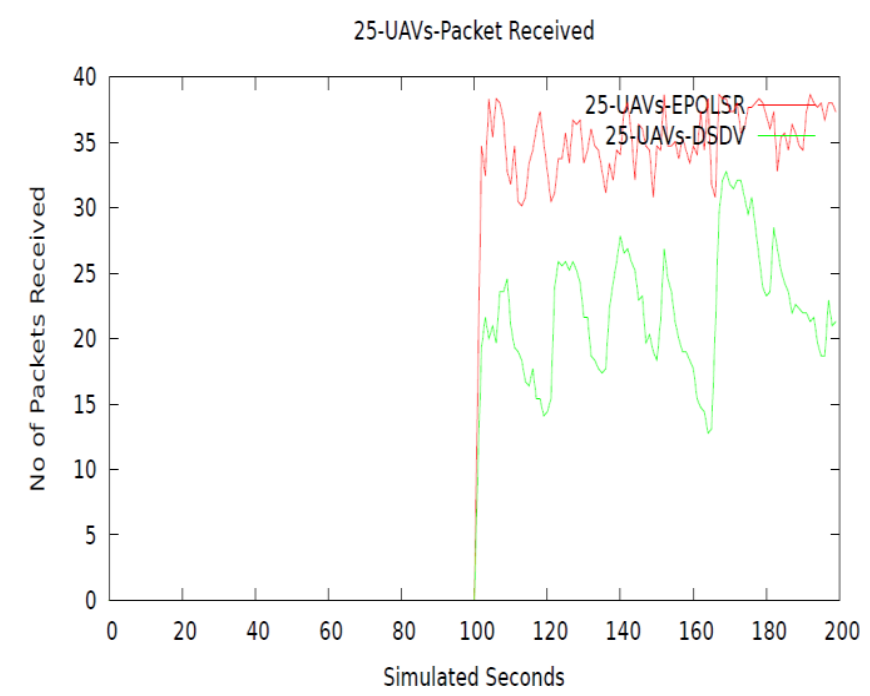

Fig. 12. 25-UAVs- No of Packet Received (kbps) using EP OLSR vs. DSDV.

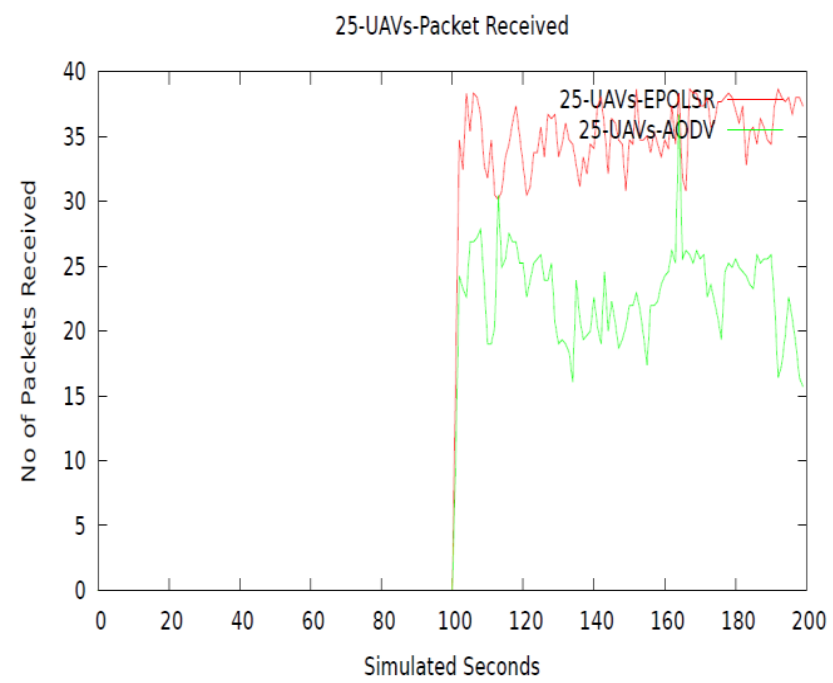

Fig. 13. 25-UAVs- No of Packet Received (kbps) using EP OLSR vs. AODV.
The results show that, in the ns- 3 environment, the EP OLSR No of Packet Received is $38 \mathrm{Kbps}$, and on the other hand, we have observed DSR No of Packet Received is 32 Kbps, as depicted in Fig. 14. Overall, the performance of the EP OLSR protocol is found better when compared with DSR.

The results show that the performance of routing protocols concerning no of the packet received in the ns-3 environment as above depicted Fig. 15. The No of Packets Received in the EP OLSR is $38 \mathrm{Kbps}$, the No of Packets Received in OLSR is $32 \mathrm{Kbps}$, the No of Packets Received in DSDV is $25 \mathrm{Kbps}$, the No of Packets Received in AODV is $26 \mathrm{Kbps}$, and the No of Packets Received in DSR is $30 \mathrm{Kbps}$. Overall, it has been observed that the performance of the EP OLSR protocol is better when compared to OLSR, DSDV, AODV, and DSR.

The results show that, in the ns-3 environment, the EP OLSR throughput is $38 \mathrm{Kbps}$, and on the other hand, we have observed OLSR throughput is $31 \mathrm{Kbps}$, as depicted in Fig. 16. Overall, the performance of the EP OLSR protocol is found better when compared with OLSR.

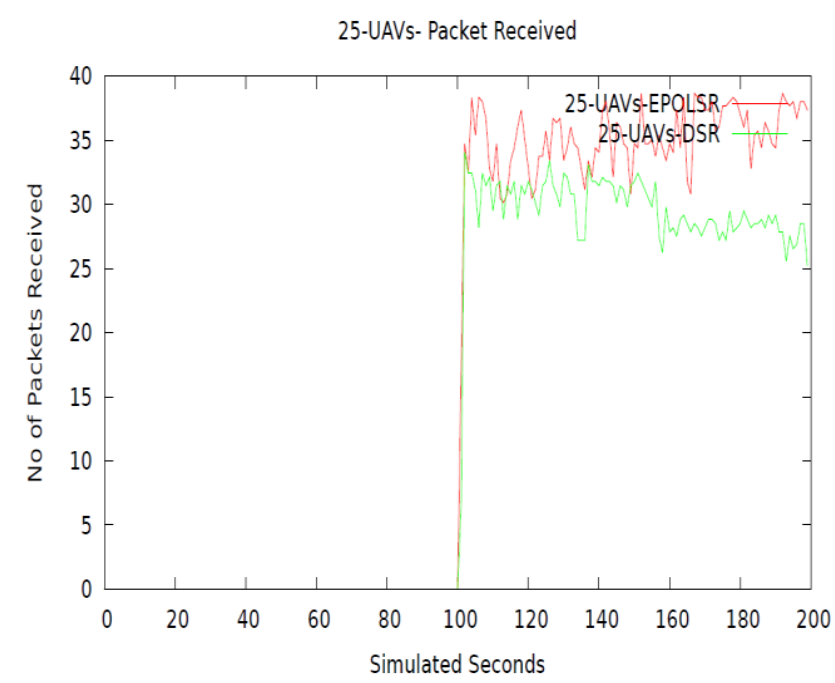

Fig. 14. 25-UAVs- No of Packet Received (kbps) using EP OLSR vs. DSR.

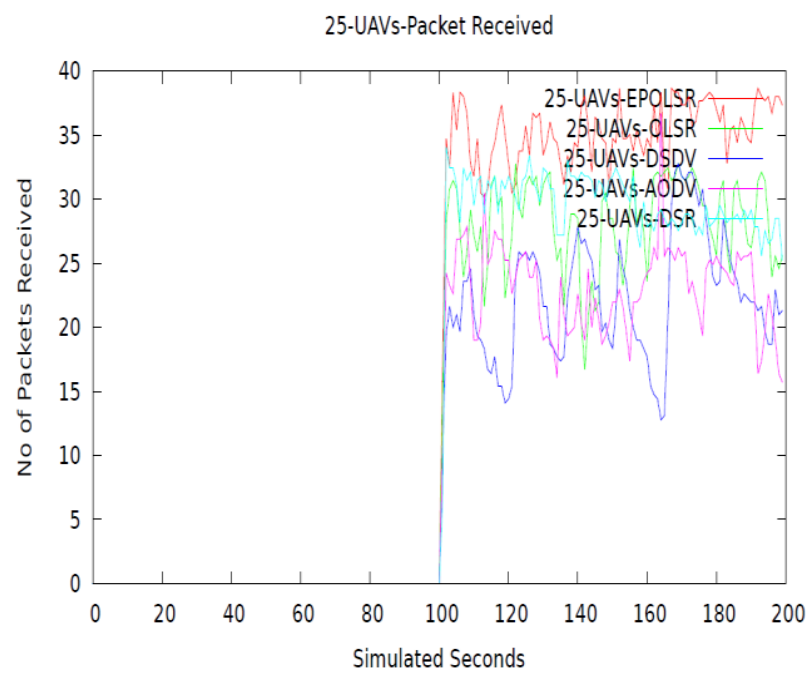

Fig. 15. 25-UAVs- No of Packets Received (kbps) using EP OLSR vs. OLSR, DSDV, AODV, and DSR 


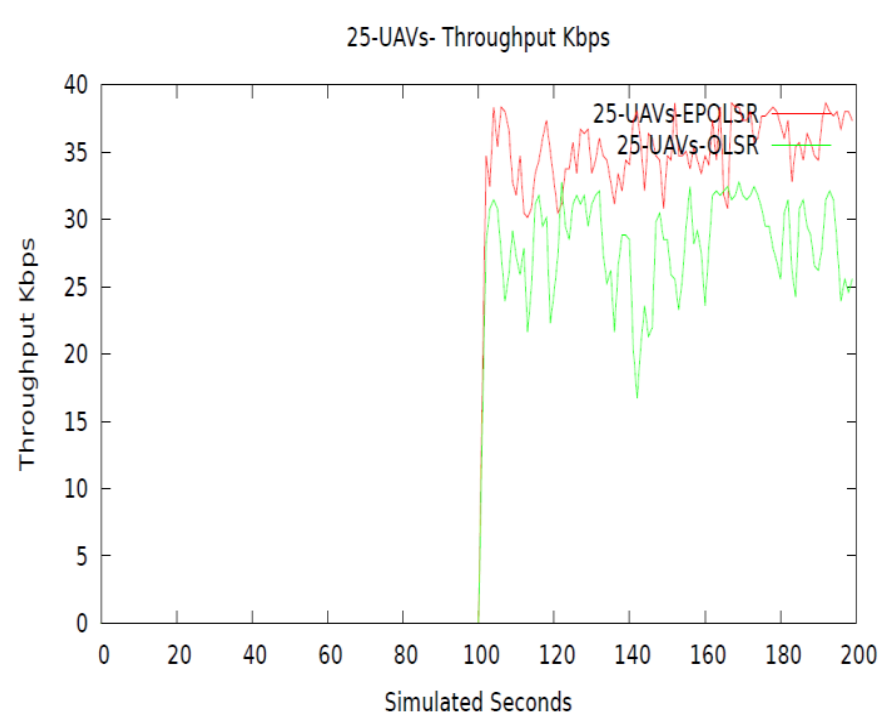

Fig. 16. 25-UAVs- throughput (kbps) using EP OLSR vs. OLSR.

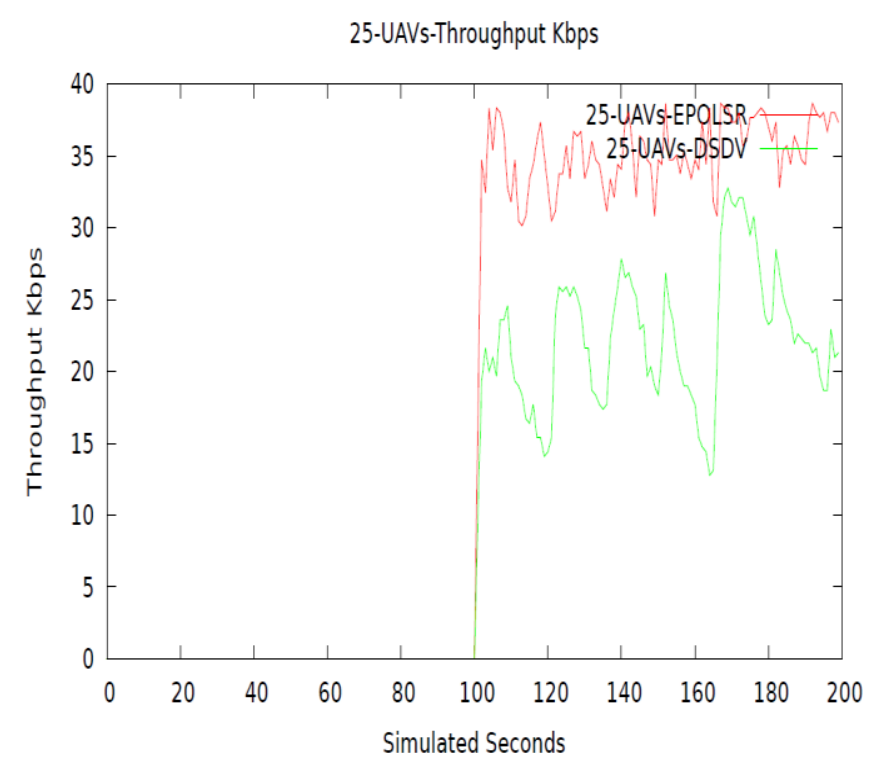

Fig. 17. 25-UAVs- throughput (kbps) using EP OLSR vs. DSDV.

The results show that, in the ns-3 environment, the EP OLSR throughput is $38 \mathrm{Kbps}$, and on the other hand, we have observed the DSDV throughput is $25 \mathrm{Kbps}$, as depicted in Fig. 17. Overall, the performance of the EP OLSR protocol is better when compared with DSDV.

The results show that, in the ns-3 environment, the EP OLSR throughput is $38 \mathrm{Kbps}$, and on the other hand, we have observed AODV throughput is $26 \mathrm{Kbps}$, as depicted in Fig. 18. Overall, the performance of the EP OLSR protocol is better when compared with AODV.

The results show that, in the ns-3 environment, the EP OLSR throughput is $38 \mathrm{Kbps}$, and on the other hand, we have observed DSR throughput is $30 \mathrm{Kbps}$, as depicted in Fig. 19. Overall, the performance of the EP OLSR protocol is better when compared with DSR.

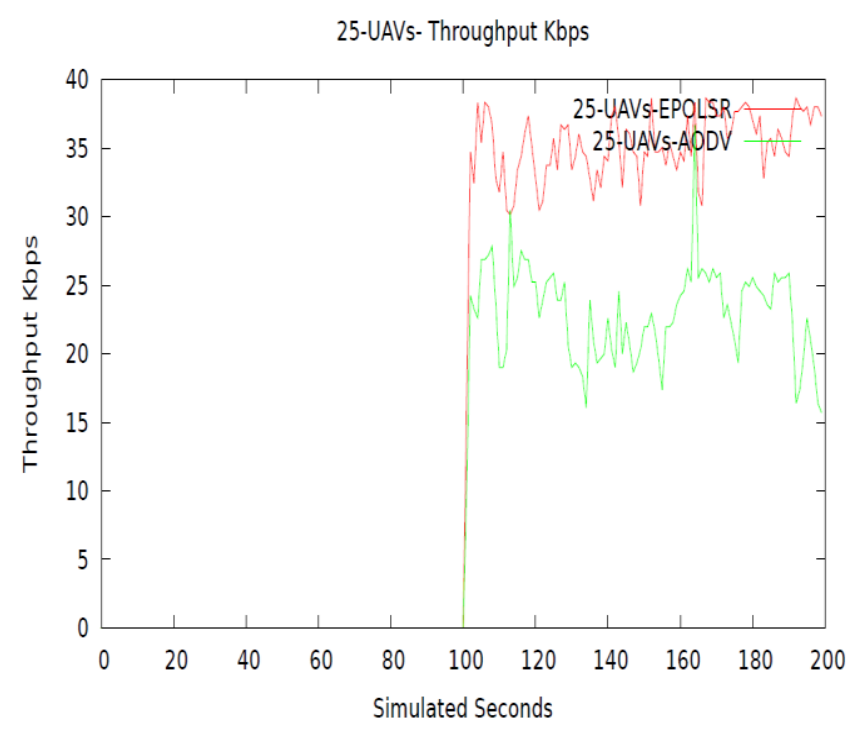

Fig. 18. 25-UAVs- throughput (kbps) using EP OLSR vs. AODV.

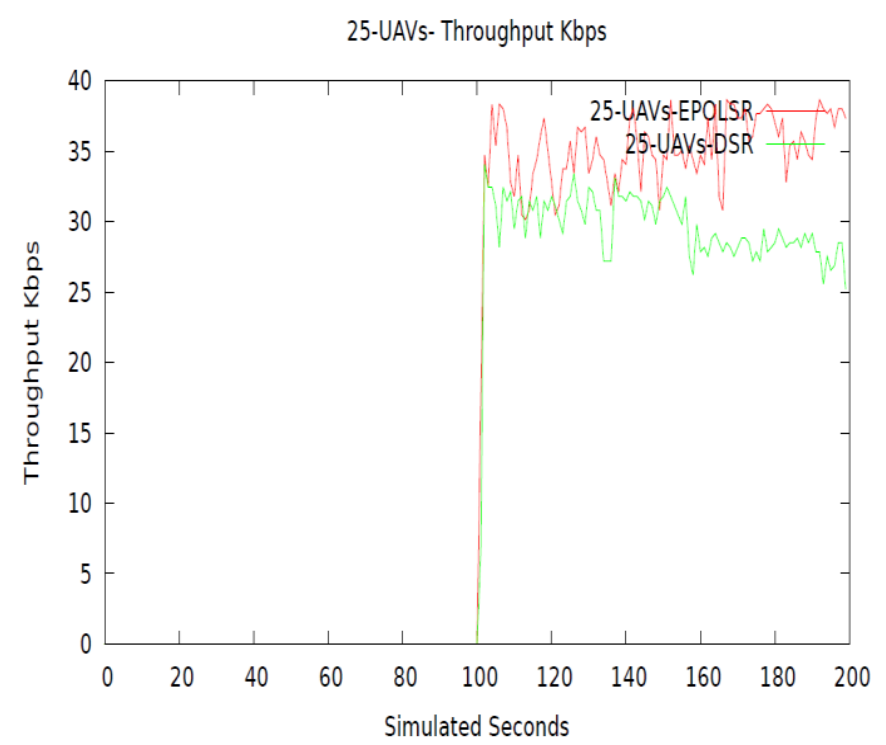

Fig. 19. 25-UAVs- throughput (kbps) using EP OLSR vs. DSR.

The results show that, in the ns-3 environment, the EP OLSR throughput is $38 \mathrm{Kbps}$, OLSR throughput is $32 \mathrm{Kbps}$, DSDV throughput is $25 \mathrm{Kbps}$, AODV throughput is $26 \mathrm{Kbps}$, on the other hand, DSR throughput is $30 \mathrm{Kbps}$, as depicted in Fig. 20. Overall, it has been observed that the performance of the EP OLSR protocol is better when compared to OLSR, DSDV, AODV, and DSR.

The results show that, in the ns-3 environment, the EP OLSR Packet Delivery Ratio is 99\%, OLSR Packet Delivery Ratio is $96 \%$, DSDV Packet Delivery Ratio is 83\%, AODV Packet Delivery Ratio is 93\%, on the other hand, DSR Packet Delivery Ratio is $91 \%$, as depicted in Fig. 21. Overall, it has been observed that the performance of the EP OLSR protocol is better than OLSR, DSDV, AODV, and DSR. 


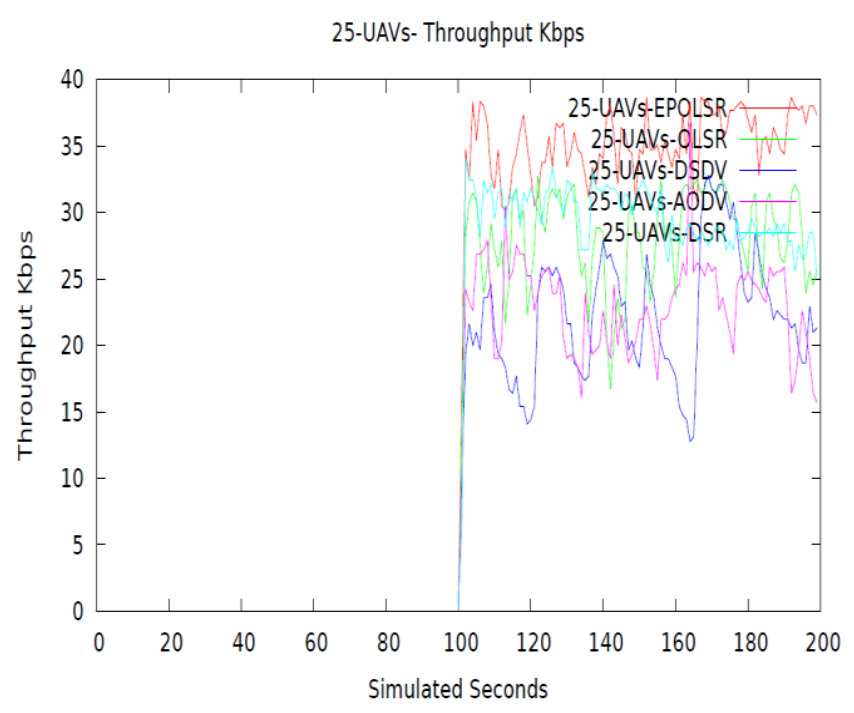

Fig. 20. 25-UAVs- throughput (kbps) $u$ sing EP OLSR vs. OLSR, DSDV, AODV, and DSR

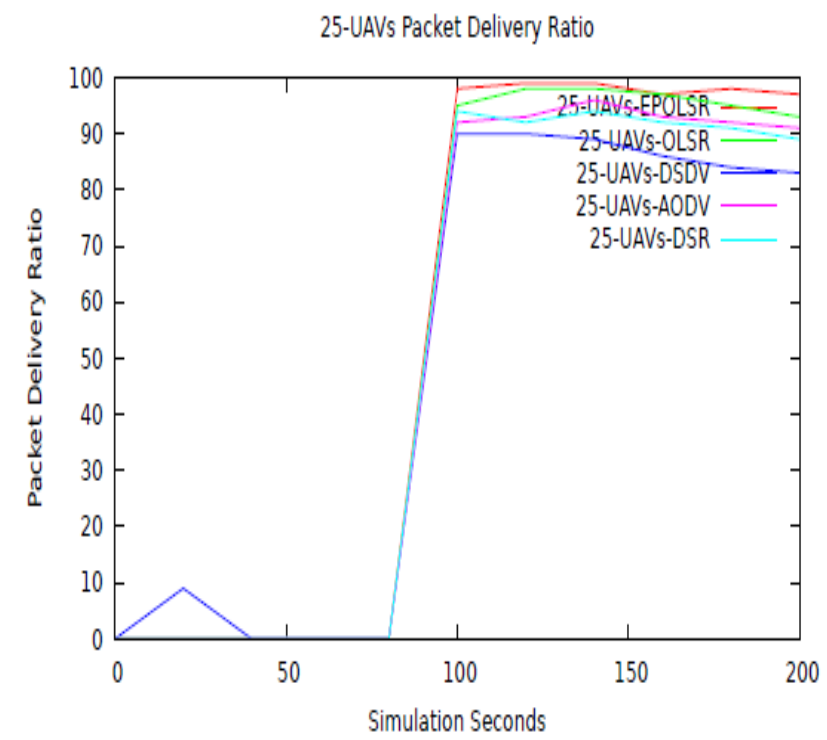

Fig. 21. 25-UAVs- Packet Delivery Ratio using EP OLSR vs. OLSR, DSDV, AODV, and DSR.

\section{CONCLUSION}

In this research, the UAVCN scenario was developed by using the NS-3. We have simulated 25 UAV nodes and implemented the proposed modified approach of routing, which is termed as EPOLSR. After this, we have evaluated the existing routing protocols with a new approach. In this paper, we have considered and used the received signal power strength (RSPS) by using an algorithm that predicts the link breakage time before route failure. And make the nodes intelligent through hello messages to ensure communication by changing route before failure. However, this method is using the interpolation method and updating the neighbor tables by power information. At the reception node, the signal power strength and time calculated for the next link breakage time by using the interpolation method. We have implemented the proposed technique by modifying the protocol OLSR. The extended protocol termed EPOLSR, which efficiently using the signal power strength and time and increasing the performance of UAVCN. The extended protocol implemented by using the research tool. The metrics received rate, no of received packets, throughput, and packet delivery ratio (PDR) are considered for evaluation. We have examined the proposed EPOLSR with existing routing protocols. It has been observed that the modified protocol performs better concerning all existing evaluated routing approaches.

\section{ACKNOWLEDGMENT}

This work has been carried out at SZABIST for the degree of Ph.D. I am very thankful to the administration of SZABIST for providing me the resources to complete this research. Moreover, I am pleased to the reviewers for their comments and grateful to the editor of IJACSA for providing the platform to publish the manuscript.

\section{REFERENCES}

[1] I. Bekmezci, O.K. Sahingoz, and Ş. Temelm, "Flying ad-hoc networks (FANETs): A survey." Ad Hoc Networks, 11(3), pp.1254-1270, 2013.

[2] H. Shakhatreh, A.H. Sawalmeh, A. Al-Fuqaha, Z. Dou, E. Almaita, I. Khalil, N.S. Othman, A. Khreishah, and M.Guizani, "Unmanned aerial vehicles (UAVs): A survey on civil applications and key research challenges." IEEE Access, 7, pp.48572-48634, 2019.

[3] L. Gupta, R. Jain, and G. Vaszkun, "Survey of important issues in UAV communication networks." IEEE Communications Surveys \& Tutorials, 18(2), pp.1123-1152, 2015.

[4] M. Mozaffari, W. Saad, M. Bennis, and M. Debbah, "Efficient deployment of multiple unmanned aerial vehicles for optimal wireless coverage." IEEE Communications Letters, 20(8), pp.1647-1650, 2016.

[5] G. Gankhuyag, A.P. Shrestha, and S.J. Yoo, "Robust and reliable predictive routing strategy for flying ad-hoc networks" IEEE Access, 5, pp.643-654, 2017.

[6] H. Yang and Z. Liu, "An optimization routing protocol for FANETs." EURASIP Journal on Wireless Communications and Networking, 2019:120, pp. 1-8, 2019.

[7] Z. Zheng, A.K. Sangaiah, and T. Wang, "Adaptive communication protocols in flying ad hoc network." IEEE Communications Magazine, 56(1), pp.136-142, 2018.

[8] K. Singh, and A.K. Verma, "Experimental analysis of AODV, DSDV and OLSR routing protocol for flying adhoc networks (FANETs)." In IEEE International Conference on Electrical, Computer, and Communication Technologies (ICECCT) (pp. 1-4), 2015.

[9] M.A. Khan, R. Hamila, M.S. Kiranyaz, and M Gabbouj, "A Novel UAV-Aided Network Architecture Using Wi-Fi Direct." IEEE Access, Vol.7, pp. 67305- 67318, 2019.

[10] I. Mahmud, and Y.Z. Cho, "Adaptive Hello Interval in FANET Routing Protocols for Green UAVs” IEEE Access, 7, pp.63004-63015, 2019. 